\title{
Différence des sexes ou distinction sexe/genre
}

Jean-Baptiste Marchand

Aux Etats-Unis, l'introduction de la notion de genre, il y a plus de 50 ans, a été une véritable révolution de l'étude de la différence des sexes. Grâce à ce terme défini comme un sexe psychosocial, les caractères masculins/féminins acquièrent une certaine indépendance par rapport au sexe mâle ou femelle limité au biologique. Cette distinction entre le sexe et le genre entraine un nouveau développement de l'étude de la différence des sexes dès lors conçue non uniquement selon le modèle binaire homme/femme mais selon deux catégories, ou dimensions : biologique et psychosociale. En effet, cette dichotomie sexe/genre permet d'isoler et de clarifier la possible présence de féminité chez l'homme ou de masculinité chez la femme.

En France, l'utilisation du genre est plus mitigée. Il y a tout d'abord un souci quant à la traduction du terme gender par celui de genre. Ce dernier ne correspond pas aux caractères masculins/féminins. Son sens est beaucoup plus large. Ainsi, pour certains «non-avertis », le genre n'a aucun rapport avec la différence des sexes. Parallèlement, les auteurs et chercheurs restent divisés car cette nouvelle perspective sexe/genre ne va pas de soi et n'est pas impartiale. On lui reproche donc d'exercer une influence et de biaiser l'étude de la différence des sexes. Le genre opposé au sexe effacerait le sexe. Dès lors, on peut constater que certains auteurs acceptent et intègrent le genre sans réserve. D'autres le refusent de manière plus ou moins radicale pour revenir au sexe. Et une dernière position est d'accepter et d'utiliser le genre tout en restant critique et en poursuivant la réflexion quant à sa définition et son influence.

Au contraire, dans les pays anglophones, le genre et sa distinction du sexe ont connu un grand succès et se sont imposés dans toutes les disciplines quelques soient les domaines, les approches ou les milieux. Ils sont devenus universels. Or, il est impossible de ne pas constater que corrélativement à cette expansion du recours au terme de genre, celui-ci a subi des modifications jusqu'à prendre des sens très différents, voire opposés, selon les auteurs. 
Prenant ces remarques comme point de départ, il semble nécessaire de revenir sur les cinquante années d'étude du genre, d'exposer son origine et de présenter les travaux majeurs, réflexions et critiques qui ont suivi l'introduction de ce concept.

\section{La distinction entre le sexe et le genre : l'émergence d'un concept et décentration du sexe}

\subsection{John Money : primauté du genre psychosocial sur le sexe biologique}

La notion de genre et sa distinction du sexe émergent en 1955 à partir des travaux de John Money ${ }^{1}$. Ce psychologue américain prend en charge des enfants souffrant d'ambiguïtés des organes génitaux au John Hopkins Hospital de Baltimore et remarque qu'une grande majorité de ces enfants (100 sur 105 en $1957^{2}$ ) adopte le sexe qui leur a été assigné à la naissance et dans lequel ils ont été éduqués.

Partant de cette première constatation, Money pense nécessaire de distinguer le sexe biologique mâle ou femelle du genre masculin ou féminin. Ces observations démontrent que le genre n'est pas inné. Au contraire, ce serait une empreinte psychologique acquise par l'apprentissage et l'expérience pouvant surpasser le sexe biologique quant au sentiment d'appartenance de l'individu à un sexe. Money conçoit alors le genre comme psychosocial et met en avant l'importance de l'éducation dans l'acquisition des comportements masculins et féminins qu'il nomme gender role, rôle de genre. Selon lui, le genre peut prendre le dessus sur le sexe puisque les enfants qu'il suit ne remettent pas en question le genre dans lequel ils ont été éduqués, même si celui-ci est en contradiction avec leur sexe.

\subsection{Robert J. Stoller : le genre en psychanalyse}

Dans la continuité de Money, le psychiatre, psychanalyste, R. J. Stoller ${ }^{3}$ reprend cette distinction entre le sexe et le genre.

Stoller travaille comme Money sur les conséquences psychiques de l'ambigüité sexuelle génitale dans la constitution de l'identité. Par la suite, il orientera ses travaux vers le

\footnotetext{
1 Money J. Hampson J. G. \& Hampson J. L. (1955), Hermaphrodism: Recommandations concerning assignement of sex, change of sex, and psychologic management, Bulletin of John Hopkins Hospital, 97, 284-300.

${ }^{2}$ Money J., Hampson J. G. \& Hampson J. L. (1957), Imprinting and the establishement of gender role, Archives of neurology and psychiatry, 77, 333-336.

${ }^{3}$ Stoller R. J. (1968), Sex and gender, vol. 1, New York, Science House, trad. franc. Recherches sur l'identité sexuelle, Paris, Gallimard, 1978.
} 
transsexualisme et le trouble de l'identité de genre chez l'enfant. L'identité de genre est dès lors étudiée, chez des patients enfants et adultes souffrant de contradictions entre leur sexe anatomique et leur sentiment d'appartenance à un sexe. Mais à la différence des patients intersexués, ceux-ci ne présentent aucune anomalie biologique.

Toutefois, son approche analytique et ses consultations avec une population différente de Money l'amènent à revisiter la notion de genre. Les troubles de «l'identité » de genre qu'il rencontre chez des adultes transsexuels et chez des enfants ne peuvent être expliqués selon la théorie de Money, à savoir par l'éducation et l'apprentissage. Avec Stoller, le genre est dès lors remanié et passe du rôle comportemental construit par l'éducation à l'identité acquise dans les relations précoces à la mère puis au père.

Se référant à la bisexualité psychique, il postule que tout individu hérite des identifications féminines et masculines de par sa relation aux parents des deux sexes lors du complexe d'œdipe. Pour lui, le sujet serait dans un mélange de masculin et de féminin. Une femme pourrait se sentir plus ou moins masculine sans remettre en cause son sentiment d'être femme et inversement pour l'homme. Si dans la plupart des cas l'identité de genre et le sexe coïncident, il peut aussi y avoir une discordance pouvant mener à la souffrance que Stoller nomme dysphorie de genre et dont l'extrême serait le transsexualisme.

Ainsi, pour lui cette distinction est nécessaire car la binarité du sexe mâle/femelle est insuffisante à rendre compte de la complexité du genre dans le rapport entre le masculin et le féminin chez l'individu.

Stoller élabore une théorie de l'identité de genre ${ }^{4}$. Selon lui, le noyau d'identité de genre est sous l'influence de cinq facteurs qui constitueront l'identité de genre définitive du nourrisson vers ses deux ans. Le premier facteur est la nature des organes génitaux du nourrisson ce que Stoller nomme la force biologique bien qu'il ne s'agit que de l'apparence anatomique. Celle-ci va directement influencer les deux facteurs suivants que sont l'assignation du sexe à la naissance et les attitudes des parents vis-à-vis du sexe du nourrisson. Entrent alors en jeu, les phénomènes biopsychiques qui sont au centre de la théorie de Stoller. Ces phénomènes renvoient à la relation précoce mère-bébé. Selon Stoller, c'est une relation

\footnotetext{
${ }^{4}$ Stoller R. J. (1985), Presentations of gender, New Haven-London, Yale University Press, trad. franç., Masculin ou feminine?, Paris, PUF, 1989.
} 
symbiotique, peau à peau, comme un cordon ombilical psychique, un état de fusion aconflictuelle dans laquelle l'identité de genre féminine de la mère va infiltrer, imprégner le noyau d'identité de genre du bébé et induire chez ce dernier une protoféminité. Au sein de cette relation, le bébé quelque soit son sexe est orienté vers le féminin par une identification première à la mère. Le garçon aura alors à se défaire de cette relation, par une protestation virile, aidé par le père qui va briser cette symbiose et s'imposer à la fois comme tiers et comme modèle auquel s'identifier. Pour la fille, le père doit comme pour le garçon briser cette relation fusionnelle mais aussi devenir un objet de désir. Puis, interviendrait comme dernier facteur, le Moi corporel en développement correspondant au vécu et aux autoperceptions par le bébé de son corps sexué. Toutefois, Stoller relativise l'importance de ce dernier facteur puisqu'il garde à l'esprit les conclusions de ses travaux et celles de Money qui montrent que même lorsque l'anatomie est défectueuse, l'individu développe une identité de genre concordante avec l'assignation du sexe et l'éducation.

Si Stoller reste celui qui a introduit la distinction sexe/genre en psychanalyse, on ne peut omettre certaines remarques et critiques quant à sa théorie. En effet, la théorie stollerienne remet en cause certains fondements centraux de la théorie analytique «classique ». Premièrement dans sa théorie, le genre devenu central vient détrôner la bisexualité psychique, l'œdipe, la castration et la différence des sexes dans la phase phallique. De même, elle ne laisse pas de place à la réalité fantasmatique et au conflit intrapsychique inconscient. Au contraire, Stoller postule une aconflictualité dans la relation mère/bébé. Par cela, sa théorie devient plus psychologique que psychanalytique. Deuxièmement, la théorie de l'identité de genre comporte des confusions et des raccourcis gênants. D'une part, Stoller semble considérer la symbiose et l'identification comme équivalentes, ce qui est critiquable. D'autre part, sa conception de la symbiose est suspecte, pour ne pas dire contradictoire. Elle serait à la fois totale : l'enfant ne se distinguerait pas de sa mère ; ce qui explique la force de l'empreinte. Mais, elle serait, en même temps, restreinte au genre : il est possible que l'enfant conserve le genre féminin bien qu'il se soit différencié de sa mère. Puis pour finir, il convient de souligner que l'identification primaire à la mère ne devrait pas être confondue avec une identification à la femme et à la féminité. Ainsi, les postulats clés de la théorie de Stoller que sont la symbiose, l'aconflictualité et la protoféminité portent bien plus à controverse qu'il ne peut y paraître au premier regard. 


\subsection{Milton Diamond : organogénèse du genre, le sexe du cerveau}

Dès la fin des années 50, la distinction sexe/genre n'est plus remise en question et connait un grand succès dans les pays anglo-saxons. Mais le genre, s'il est accepté, devient aussi sujet de controverse. Ainsi, bien qu'étant un des plus grands opposants à la théorie de Money, Milton Diamond professeur d'anatomie et de biologie de la reproduction à l'université de Hawaï, intègre la différence entre le sexe et le genre à ses travaux.

Le désaccord qui oppose Diamond à Money porte sur le caractère inné ou acquis du genre. Diamond ${ }^{5}$ conteste que le biologique soit relégué au second plan. Selon lui, le genre a une origine organique, hormonale et surtout cérébrale. L'identité de genre sociale ne peut être sans lien avec l'identité sexuelle du cerveau. La subtilité vient du fait que Diamond différencie cette identité sexuelle du sexe génital. L'identité sexuelle est différenciée du trop simple sexe chromosomique $\mathrm{XX}$ ou $\mathrm{XY}$ et des organes génitaux.

Les premiers travaux de Diamond sont basés sur des expérimentations sur des animaux. Par la suite, il travaille comme Money et Stoller avec des patients intersexués et avec des patients transsexuels. Il considère d'ailleurs ces derniers comme des intersexués ayant un corps et des organes génitaux d'un sexe mais un cerveau de l'autre.

La distinction de Diamond ${ }^{6}$ entre identité sexuelle et identité de genre n'est ni simple, ni évidente. Pour lui, tout individu vit avec deux visions simultanées de lui-même ; l'identité sexuelle qui est interne et privée ; et l'identité de genre qui est extérieure, publique et sociale. La première est organisée durant la période prénatale par les forces génético-endocriniennes et se poursuivra avec le développement. La seconde, se rapportant à la manière dont la personne est reconnue et considérée en société, se développe par les expériences postnatales, sous l'influence de la première.

L'enfant nait d'une part avec une prédisposition psychosexuelle biologique déterminée par l'héritage génético-endocrinien et d'autre part, avec une orientation vers certains schèmes sexuels et modèles de genre qui pourront plus ou moins s'exprimer selon les mœurs sociales et culturelles et le degré de tolérance que ces dernières autorisent.

\footnotetext{
${ }^{5}$ Diamond M. (1965), A critical evaluation of the ontogeny of sexual behavior, Quarterly review of biology, 40, 147-173.

${ }^{6}$ Diamond M. (2000), Sex and gender: same or different?, Feminism \& Psychology, 10 (1), 46-54.
} 
Dès ses premiers travaux, Diamond réfute la théorie du genre psychosocial acquis par l'apprentissage de Money et cherche à prouver que l'identité de genre est innée. Pour se faire, il entreprend de nombreuses études critiques de la théorie et des travaux de Money et essaye, en parallèle, de contacter les patients de Money. Cette dernière démarche le mènera à rencontrer David Reimer ${ }^{7}$, patient pour lequel la prise en charge thérapeutique par Money a été un échec (même s'il faut différencier cet échec thérapeutique des observations cliniques de Money). La publication de cet échec par Diamond ${ }^{8}$ fit un scandale et jeta le discrédit sur l'intégralité des travaux de Money. Diamond voit dans cet échec la confirmation de l'importance de la force du biologique génético-endocrinien sur l'acquisition par l'éducation.

\subsection{Le genre en ethnologie : du troisième sexe au troisième genre, le genre comme construction culturelle}

Parallèlement à ce débat, les ethnologues reprennent le concept genre et par celui-ci revisitent notamment les études du troisième sexe. Le genre perd son caractère psychologique et est défini en tant que construction sociale et culturelle en opposition avec la finitude du corps sexué. Les études du troisième sexe/genre ${ }^{9}$ exposent que dans certaines peuplades, des individus adoptent le rôle de l'autre sexe, sans changements corporels.

Par exemple, chez les Inuits ${ }^{10}$, la déclaration du sexe de l'enfant est entrainée par des circonstances liées aux traditions de la culture. Indépendamment de la nature des organes génitaux de l'enfant, la mère assigne un genre au nourrisson par le choix du nom d'un ancêtre selon son vécu de la grossesse, selon ses rêves et selon la nécessité d'un équilibre de l'unité familiale $^{11}$. L'enfant est alors déclaré et élevé dans le rôle du sexe opposé. Il prend les habits et adopte les comportements et les rôles des membres du sexe opposé et ce jusqu'au moment de la puberté (premières règles pour les filles et premier abattage du gibier pour les garçons) où il est réintégré dans le genre concordant avec son sexe biologique. Après cette

\footnotetext{
${ }^{7}$ Reimer, alors qu'il était encore un nourrisson, a dû subir une ablation du pénis suite à un accident chirurgical. Money avait alors proposé à ses parents de le réassigner dans le genre féminin pensant lui éviter la vie difficile d'un garçon puis d'un homme sans pénis. Reimer n'a jamais accepté cette réassignation.

${ }^{8}$ Diamond M. \& Sigmundson H. K. (1997), Sex Reassignment at Birth: A Long Term Review and Clinical Implications, Archives of Pediatrics and Adolescent Medicine, 151, 298-304.

${ }^{9}$ Herdt G. (1994), Third Sex, Third Gender, Beyond sexual dimorphism in culture and history, New York, Zone Books.

${ }^{10}$ Saladin d'Anglure B. (1986), Du fœtus au chamane : la constitution d'un troisième "sexe" inuit, Etudes/Inuit/Studies, 10, 1-2, 25-113.

${ }^{11}$ L'enfant peut être déclaré d'un genre opposé à son sexe pour que la famille soit composée d'un père, d'une mère et d'un garçon et d'une fille.
} 
réassignation, après avoir vécu tour à tour dans les deux sexes, ces enfants gardent un statut particulier de chaman au sein de leur communauté.

De même, chez les amérindiens, les berdaches ${ }^{12}$ vivent dans le genre opposé à leur sexe. Les berdaches sont des hommes devenus femme suite à une révélation spirituelle (un rêve ou une vision) ou lors d'un état de transe après lequel leur statut social de «femme » (de berdache) sera révélé et établi. Les berdaches s'habillent en femme, adoptent des attitudes féminines et réalisent des tâches habituellement réservées aux femmes. Comme chez les Inuits, les berdaches ont au sein de leur communauté un statut sacré avec des pouvoirs supranaturels notamment de guérisseur.

Ils existent donc des cultures qui disposent d'un statut social pour des personnes se situant en-dehors de la dichotomie homme/femme ou permettant à ces personnes de vivre dans le rôle du sexe opposé. Toutefois, il est compliqué de distinguer la part d'un statut masculin/féminin de celle d'un statut plutôt socioculturel notamment sacré. Ainsi, deux questions restent posées. Celle de savoir comment sont reconnus ces individus par les autres membres de leur communauté ; et celle du ressenti subjectif de ces individus quant à leur genre/sexe. Dès lors, pour les membres de la communauté comme pour ces individus euxmêmes, on ne peut être certain que ces personnes se sentent et soient considérés sans équivoque comme appartenant au sexe opposé (homme ou femme), ou bien comme des sujets vivants dans un genre opposé à leur sexe anatomique reconnu (homme féminin ou femme masculine).

\section{Tournant social et politique de la conception du genre : féminisme, gender studies et théorie queer}

\subsection{Brève présentation historique des mouvements sociopolitiques du genre}

Présenter ce tournant n'est pas une tâche aisée. Le sexe est mis de côté et le genre passe au premier plan. Il y a à la fois un tournant et un vaste élargissement du recours à la notion de genre qui se font à la fois dans deux milieux : universitaires et militants ; et dans deux mouvements : féministe et gay et lesbien ${ }^{13}$. Ainsi, avant de présenter les travaux de

\footnotetext{
${ }^{12}$ Roscoe W. (1987), Bibliography of berdache and alternative gender roles among North American Indians, Journal of Homosexuality, 14-3 et 4, 81-171.

${ }^{13}$ Il est préférable d'utiliser les termes gay et lesbien plutôt qu'homosexuel car cette nomination correspond à celui du mouvement et non à l'unique orientation sexuelle.
} 
Judith Butler qui sont la référence incontournable de cette approche sociopolitique, il semble pertinent de présenter le contexte qui précède l'écriture de Trouble dans le genre ${ }^{14}$. La multiplication des terrains et des mouvements nommés ci-dessus amène à une vague de séparatisme au sein de chaque mouvement ${ }^{15}$.

La première rupture concerne le mouvement féministe. Durant les années 70-80, une partie conservatrice des féministes rejette les lesbiennes et les transsexuels en les accusant de reproduire et/ou de coopérer avec la domination de l'homme sur la femme. Dans le même temps, des féministes lesbiennes rompent avec ce mouvement conservateur en lui reprochant son hétéronormativité qui ferait obstacle à la libération des femmes. Cette idée étant résumée par le fameux quasi syllogisme de Wittig : "la femme n'a de sens que dans les systèmes de pensée et les systèmes économiques hétérosexuels. Les lesbiennes ne sont pas des femmes. » ${ }^{16}$ C'est la naissance des gender studies.

La seconde scission a lieu dans le mouvement gay et lesbien, dans les années 80-90 lors des années sida et de l'émergence d'Act-up. Une partie de la communauté homosexuelle défavorisée et exclue (majoritairement des femmes, lesbiennes, chômeuses et issues de minorités ethniques) se désolidarise du mouvement collectif d'affirmation identitaire. C'est l'apparition de la revendication queer en opposition au straight, affirmation des minorités contre le mouvement collectif gay et lesbien accusé de s'embourgeoiser, de délaisser les minorités exclues et de reposer les bases d'un modèle d'intégration et de normalisation de l'homosexualité en référence au modèle hétérosexuel de la classe moyenne supérieure blanche. La nomination queer qui signifie bizarre est revendiquée pour se confronter au straight signifiant droit, bon, normal, sain. De même, il se produit une séparation, bien qu'elle ne soit pas totale, entre les scènes militantes et universitaires. Les champs d'investigations sont volontairement flous et pluriels car anti-identitaires. Seul l'objectif semble clairement défini à savoir bousculer les frontières établies entre homme et femme par le recours à la déconstruction et l'activisme.

\footnotetext{
${ }^{14}$ Butler J. (1990), Gender trouble : feminism and subversion of identity, New York \& London, trad. franç., Trouble dans le genre : féminisme et subversion de l'identité, Paris, La Découverte, 2005.

${ }^{15}$ Califa P. (1997), Retour de manivelle : la transphobie chez les féministes in Sex Changes, The Politics of Transgenderism, San Francisco, Cleis, trad. franc., Le mouvement transgenre, changer de sexe, 123-169, 2003.

${ }^{16}$ Wittig M. (1973), Le corps lesbien, Paris, Minuit, p76.
} 


\subsection{Judith Butler : la mélancolie de genre entre psychanalyse et politique}

C'est dans ce contexte séparatiste qu'est publié Trouble dans le genre : le féminisme et la subversion de l'identité ${ }^{17}$, l'œuvre majeure de Judith Butler. Butler est une philosophe, professeur de rhétorique et de littérature comparée à l'université de Berkeley. A ce jour, elle est l'une des figures les plus importantes de la théorie queer et des gender studies. Ces travaux sur le genre sont particulièrement novateurs tant d'un point de vue théorique que dans leur perspective d'analyser le lien entre la formation de la psyché et l'ordre politique et social.

Dans Trouble dans le genre, Butler procède à une déconstruction du genre avec des références psychanalytiques. Selon elle, la psyché se forme par l'intériorisation des normes et le genre serait la traduction culturelle de l'hétérosexualité obligatoire, fondement d'une identité normative. De même, le genre crée le corps érogène et ses désirs érotiques.

Plaçant les normes sociales au premier plan, Butler postule qu'un tabou de l'homosexualité précéderait le tabou de l'inceste et situe donc le conflit entre l'individu et la société. L'homosexualité prohibée entraine une exclusion sociale qui par transposition induit une exclusion normative du rapport au parent du même sexe, point de départ de la genèse de la mauvaise conscience. A partir de ce tabou, le genre agirait sur l'individu telle une mélancolie. L'ontogenèse du soi passerait par une mélancolie de genre. A la différence du travail de deuil qui par le processus d'introjection permet un réaménagement et le réinvestissement du désir dans un nouvel objet à aimer; dans la mélancolie, l'incorporation situe l'objet perdu dans le corps et entraine une disparition de la conscience du sujet. Dans la mélancolie, le désir est perdu avec l'objet. La perte concerne l'objet et le but de la pulsion. Le désir tout entier disparait avec l'objet. Cette perte amène une fixation de la pensée dans laquelle le renoncement et le réaménagement deviennent impossibles. Dans la mélancolie de genre, en réaction au tabou de l'homosexualité, le sujet serait amputé d'une partie érogène de son corps et perdrait avec celle-ci le désir érotique homosexuel.

Parce que, pour Butler, le genre et l'identité se constituent par des actes, la libération de la mélancolie de genre peut se faire par des actes. Il faut défaire le genre par la capacité d'agir (agency). Il faut exprimer son identité personnelle par les actes. Le genre est une mascarade, un jeu au sens théâtral et peut donc être rejoué et surtout dépassé en le parodiant.

\footnotetext{
${ }^{17}$ Butler J. (1990), op. cit.
} 
Il relève de la performativité : «l'énoncé crée la chose ${ }^{18}$. Ainsi, selon elle, le travesti porte le deuil du genre, dévoile la possibilité de lui échapper et de dépasser les frontières faussement binaires induites par l'hétérosexualité. La dérision par le travestissement montre que le genre n'existe pas en-dehors de la répétition.

Bien que la mélancolie de genre semble cohérente et correctement structurée, cette théorie de Butler conserve des zones d'ombre. Sa conception du genre n'est pas clairement définie. Comme le rapporte Monique David-Ménard ${ }^{19}$, après s'être entretenue avec Butler, dans Trouble dans le genre, le genre veut à la fois dire sexe et genre. Butler relativise même la nécessité de cette distinction ${ }^{20}$. Le sexe serait lui aussi une construction culturelle à laquelle on octroie de manière erronée une immuabilité. Cette définition trop vague peut être remarquée dans Défaire le genre. Dans le chapitre, La fin de la différence sexuelle ${ }^{21}$, Butler constate le succès et l'expansion du recours à la notion de genre. Mais comme en contrepartie, elle est aussi confrontée à la torsion du concept selon les groupes et institutions qui y ont recours en tant que signifiant politique. Les queer studies s'intéressent au sexe et à la sexualité à travers des études gays et lesbiens et considèrent le genre comme relevant de l'étude de la condition de la femme et donc du féminisme. Les féministes privilégient la différence sexuelle car le genre construit socialement ne conviendrait pas à la spécificité politique du féminin. Pour les membres du Vatican, il est synonyme de sexualité identitaire renvoyant au masculin, féminin, gay, lesbien et transsexuel. Alors que pour la commission de l'ONU, le genre en tant que construction sociale est à prendre en considération dans les rôles et le statut des hommes et des femmes notamment concernant l'autonomie et les acquis obtenus par ces dernières.

D'autre part, il est nécessaire de revenir sur le postulat d'un tabou «premier » de l'homosexualité, sur l'usage de la mélancolie et sur la référence plus ambigüe qu'ambivalente à la psychanalyse. Le tabou de l'homosexualité dans la théorie de Butler se réfère aux points

\footnotetext{
${ }^{18}$ Toutefois, Butler met de côté que la valeur performative d'un énoncé dépend du contexte, du locuteur et du destinataire.

${ }^{19}$ David-Ménard M. L'intérêt pour la psychanalyse dans les travaux de Judith Butler : entretien avec Livio Boni in Revue mondiale des francophonies [en ligne].

http://mondesfrancophones.com/espaces/psyches/linteret-pour-la-psychanalyse-dans-les-travaux-de-judithbutler-entretien-avec-livio-boni/ (page créée 19 novembre 2007, consultée le 12 novembre 2010 ).

${ }^{20}$ Butler J. (1990), op. cit., p 69.

${ }^{21}$ Butler J. (2004), La fin de la différence sexuelle in Undoing gender, New York and London, Routledge, trad. franç., Défaire le genre, Paris, Editions Amsterdan, 2006, 201-231.
} 
de vue féministe et gays et lesbiens. Or, le tabou de l'homosexualité est cohérent avec le développement de la petite fille dans son rapport à sa mère, ce que Butler nomme l'homoattachement originaire. Mais la question de l'application au garçon de cet homo-attachement et donc du tabou de l'homosexualité et de la mélancolie de genre reste en suspend. De plus, le tabou de l'homosexualité implique que l'enfant prenne en compte le sexe de l'objet, du parent. Ceci n'est pas compatible avec le point de vue génétique de la psychanalyse selon lequel il ne peut y avoir homosexualité qu'après l'œdipe. Les premiers amours préœdipiens et la sexualité infantile ne peuvent être confondus avec l'homosexualité. De même, Butler ne fait pas la distinction entre les identifications primaire et secondaire. Ajoutons à cela, l'absence de référence ou la disparition de concepts essentiels comme la bisexualité psychique et l'ambivalence. De même, la théorie de Butler ne fait pas référence à l'Inconscient mais seulement à un Moi réduit à une somme d'identifications dépourvues de conflit intrapsychique. Ce dernier est en effet remplacé par le conflit de l'individu avec la société.

Concernant le recours à la mélancolie, celui-ci n'est pas cohérent avec la psychopathologie. Premièrement, Butler utilise la mélancolie pour rendre compte de la constitution du Moi alors qu'elle est au contraire une profonde remise en cause de celui-ci. De plus, ce recours cache un paradoxe. En effet, Butler choisit la mélancolie pour expliquer la perte totale du désir et du corps érogène homosexuels mais en même temps cette perte ne serait que partielle puisque ne concernant que le genre. Or, en psychopathologie, la mélancolie agit en tout ou rien. C'est la totalité de la libido qui investit le Moi qui est pris totalement dans la perte. Cette absence de processus ne peut se limiter au genre. La mélancolie de genre, si elle est théoriquement possible, n'est pas compatible avec la clinique de la mélancolie.

Reste à souligner, l'ambigüité de la position de Butler vis-à-vis de la psychanalyse. Par sa démarche, elle cherche à montrer les limites d'une théorie freudienne partiale puis elle entreprend une reconstruction autour de la mélancolie de genre. Cette démarche sème le doute. Les gender studies et la théorie queer sont plutôt réticentes voire hostiles à l'approche psychanalytique. Or, la théorie analytique et la mélancolie de genre sont présentées et examinées de manière pédagogique sans que le lecteur puisse comprendre si Butler est influencée, adhère ou s'oppose à la psychanalyse. Faut-il adhérer à une théorie psychanalytique remaniée par le tabou de l'homosexualité et la mélancolie de genre ? Ou ces 
deux fondements de la théorie de Butler amènent-ils à un rejet de la psychanalyse ? De la même manière, on retrouve ce manque de clarté ou ce refus de choisir, de trancher clairement lorsque Butler poursuit ses travaux dans des collaborations avec des psychanalystes alors qu'elle laisse entendre dans ses autres travaux plus proches des gender studies et de la théorie queer que la psychanalyse serait inutile ou erronée car trop hétéronormée et qu'elle s'opposerait donc à celle-ci.

\section{La distinction sexe/genre en psychanalyse contemporaine}

Avant de conclure, il est intéressant de présenter les points de vue d'auteurs contemporains de l'émergence et de l'expansion de la distinction sexe/genre. Je mets de côté une présentation de la théorie de la sexuation lacanienne et les travaux inspirés de celles-ci. Bien que Lacan ait lui-même parlé des travaux de Stoller et que ses successeurs s'intéressent à la théorie queer et aux gender studies ${ }^{22}$, ces travaux n'arrivent pas à aboutir à une réflexion constructive sur le genre. Ils restent dans l'impasse d'une opposition trop franche et directe comme si il y avait une incompatibilité. Succinctement, la notion de genre et toutes les théories qui en découlent, agissent au détriment du sexe (limité au Réel) et font l'erreur de confondre le pénis et le phallus, l'organe et le signifiant nécessaire à la reconnaissance symbolique de l'identité sexuelle par la parole de l'Autre (les parents). Bien que très intéressante, présenter cette critique serait en quelque sorte hors sujet puisqu'une fois le genre réfuté, il ne reste plus que le sexe et le débat perd sa légitimité.

Les deux auteurs présentés ci-dessus entreprennent une étude différente de la distinction entre le sexe et le genre. Laplanche reprend la notion de genre en psychanalyse et interroge la place qu'elle peut prendre par rapport à la distinction faite en psychanalyse entre la sexualité adulte et la sexualité infantile refoulée. Dans une perspective différente, Chiland revient sur le souci de traduction du terme gender par celui de genre et poursuit cette réflexion en se recentrant sur le terme de sexe.

\footnotetext{
${ }^{22}$ Association de la revue lacanienne, Melman C. \& al. (2007), Dossier : Sex and gender, Bulletin lacanien, 4, 4, 9-60.
} 


\subsection{Jean Laplanche : théorie de la sexualité élargie et révision du genre en psychanalyse}

Laplanche ${ }^{23}$ revisite les différents courants qui ont étudié la distinction sexe/genre et ajoute à ce couple d'opposés ce qu'il nomme le sexual. Le sexe est duel de par la reproduction sexuée et la symbolisation de la phase phallique: présence/absence, phallique/castré. Le sexual se distingue du sexe. Le sexual est pervers et polymorphe. Il est la découverte freudienne: le refoulé de la sexualité infantile condamnée par l'adulte. Il se fonde dans l'inconscient, dans le fantasme par le refoulement. Pour Laplanche, il y a une opposition entre le sexe et le sexual, entre la différence des sexes et la sexualité infantile orale, anale, paragénitale. Pour sa part, le propre du genre serait, selon Laplanche, de véhiculer des contenus (en grande partie inconscients) conceptuellement impurs touchant au sexe et à la sexualité. Le genre agirait donc en coopération avec le sexe «adulte» contre le sexual. L'opposition sexe/genre s'avèrerait être un couple d'alliés contre le sexual. Dès lors, Laplanche propose l'hypothèse que le sexual serait le résidu inconscient du refoulementsymbolisation du genre par le sexe.

Laplanche remanie le concept de genre et critique les études ethnologiques et féministes de celui-ci. Il souligne que ces dernières négligent la situation anthropologique fondamentale $^{24}$ de l'enfant. Leur position est trop adulto-centrique. Dès lors, l'énigme du genre, du masculin-féminin ne devrait pas être envisagée selon le point de vue de l'enfant qui devient adulte ou selon celui de l'adulte se remémorant l'enfant mais dans la simultanéité de l'enfant, en présence de l'adulte, s'interrogeant sur cette différence chez ce dernier. De même, il accepte que le genre soit social car le genre est assigné. Toutefois, il demande une certaine prudence quant à cette définition du genre. Pour lui, le genre n'est pas social dans le sens du rapport de l'individu à la société ou à la culture. Il l'est dans le sens du rapport de l'enfant aux petits groupes de socii que sont ses parents et ses proches, au sens du socius de la préhistoire personnelle de l'enfant. Ce n'est pas la société qui assigne le genre. Ce sont les proches.

Pour Laplanche, il y a une précession du genre sur le sexe, du social sur l'anatomique (descriptif et perceptif, différent du biologique) par le primat de l'Autre qui s'exprime dans

\footnotetext{
${ }^{23}$ Laplanche J. (2007), Le genre, le sexe, le sexual in Sexual, la sexualité élargie au sens freudien, Paris, PUF, 153-193.

${ }^{24}$ Relation universelle entre l'enfant qui n'a pas d'inconscient génétiquement programmé et un adulte habité par un inconscient.
} 
l'assignation. Toutefois, le genre n'organise pas le sexe. Au contraire, il serait organisé par lui dans la séduction généralisée. La théorie de la séduction généralisée explique l'origine de la pulsion en plaçant l'attachement comme un code véhiculé par les soins corporels au sein de la relation parent-enfant. Elle est basée sur l'idée que la relation d'attachement est une communication. Celle-ci comporte des messages de l'autre passant par un langage préconscient-conscient conçu tel un code et du bruit de l'inconscient parental venant perturber cette communication, compromettre ces messages. Il y a simultanéité du fait que les deux protagonistes enfant-adulte sont actifs dans cette communication. L'adulte émet le message. L'enfant reçoit le message et traduit le code.

Concernant l'assignation du genre, elle se ferait par ces messages comportant le bruit de l'inconscient sexué et sexual de l'adulte. Le genre est donc acquis de façon énigmatique par ces messages et sera traduit et fixé par le sexe vers deux ans. D'autant plus, que pour Laplanche, l'adulte en présence de l'enfant est confronté à la réactivation de sa propre sexualité infantile. Il préconise donc qu'il s'agirait plutôt d'une identification «par » l'adulte que «à » l'adulte.

Laplanche résume son point de vue par la triade ordonnée genre-sexe-sexual : d'abord, le genre social ; ensuite, le sexe de la différence des sexes ; puis, le sexual : sexualité infantile condamnée par l'adulte, résidu du refoulement du genre par le sexe.

\subsection{Colette Chiland : l'identité sexuée comme construction psychique}

Bien qu'étant traductrice de Stoller, Chiland ${ }^{25}$ garde un esprit critique sur la traduction du terme gender par celui de genre. Par exemple, l'expression mauvais genre ne fait aucune allusion au masculin ou au féminin. En 1985, si elle choisit d'employer le mot genre dans la traduction de Stoller, c'est pour respecter la conceptualisation de ce dernier. En 1994, elle souligne que la distinction sexe/genre de Money amène une confusion entre identité sexuée (sentiment et croyance d'appartenance à un sexe), conduites manifestes liées aux stéréotypes sociaux et vie érotique ${ }^{26}$. Après la traduction de Stoller, elle s'intéresse à la naissance de l'identité sexuée chez les enfants qu'elle suit et s'oriente vers le transsexualisme chez l'adulte avec le Dr Le Breton, pionnier de la prise en charge de ce syndrome à Paris.

\footnotetext{
${ }^{25}$ Chiland C. (1988), Enfance et transsexualisme, Psychiatrie de l'enfant, XXXI, 2, 313-373, p 333.

${ }^{26}$ Chiland C. (1994), Homosexualité féminine et identité sexuée, Revue française de psychanalyse, 1, 147-156, p 150.
} 
A partir de cette remarque, elle propose plutôt de se référer à une déclinaison du sexe ${ }^{27}$ selon les trois axes : biologique, psychologique et social ; et de distinguer pour chacun de ces axes deux perspectives que sont la sexuation et la sexualité, le sexué et le sexuel. A l'origine réservée à la biologie, la sexuation relève de la division de l'espèce humaine en deux sexes, le sexué marquant l'appartenance à l'un des deux sexes (homme, femme avec la nuance des incongruences intersexuées) mais Chiland utilise ce terme selon les trois déclinaisons du sexe et l'élargit donc au psychologique et au social. La sexualité est restreinte à l'attirance, au choix d'objet sexuel, à la relation érotique et à l'orientation sexuelle (hétérosexualité, homosexualité et bisexualité).

A partir de ces deux perspectives, Chiland suggère de différencier la bisexualité psychique de la bisexuation psychique ${ }^{28}$. A l'origine, la bisexualité psychique confondait à la fois l'identification aux parents des deux sexes et l'attrait aux parents des deux sexes dans les deux versants négatif et positif de la relation œdipienne. Or, la bisexuation psychique serait à envisager comme l'aspect identificatoire à distinguer de l'attirance qui resterait le propre de la bisexualité.

Selon Chiland, l'identité sexuée, le sentiment d'appartenir à l'un des deux sexes, est une construction psychique ${ }^{29}$. Elle se retrouve aux trois niveaux biologique, psychologique et social du sexe. Chiland remarque d'ailleurs que la construction psychique de l'identité sexuée est un paradigme de la constitution de la psyché entre nature et culture. Il y a un roc psychologique $^{30}$ à côté du roc biologique. Son origine prend place dans les interactions précoces de l'enfant à ses parents. La définition de l'identité sexuée par Chiland se réfère à la définition du Self de Winnicott. L'identité sexuée est la continuité d'être en tant qu'être humain individuel dans son corps et dans son sexe ${ }^{31}$. Cette identité se forme de manière irréversible dans les deux premières années de la vie. Bien qu'il n'y ait pas une essence de l'identité sexuée, Chiland précise que cette construction psychique est d'emblée sexuée par l'interaction bébé-parent. L'enfant a un sexe dans la tête de ses parents avant même sa

\footnotetext{
${ }^{27}$ Chiland C. (1995), De l'essence du féminin, Psychologie clinique et projective, 1, 2, 143-160, p 152.

${ }^{28}$ Chiland C. (1995), Ibid., p 155.

${ }^{29}$ Chiland C. (2003), Nouveaux propos sur la construction de l'identité sexuée, Journal de psychanalyse de l'enfant, 33, 105-122.

${ }^{30}$ Braconnier A. (2005), Entretien avec Colette Chiland, Carnet psy, 97, 2, 35-41.

31 Chiland C. (2005), Problèmes posées aux psychanalystes par les transsexuels, Revue française de psychanalyse, 69, 1, 2, 563-577.
} 
naissance. Elle ne devient pas sexuée dans un second temps, après une identité neutre ou indifférenciée.

\section{Conclure après plus de $\mathbf{5 0}$ ans de travaux sur le genre}

En 1920, Freud ${ }^{32}$, à partir de ses travaux sur 1'homosexualité, soulignait déjà que confondre les caractères sexuels somatiques, les caractères sexuels psychiques et le choix d'objet étaient une erreur, et appelait donc à les distinguer. Bien qu'elles soient différentes, il est impossible de ne pas faire le rapprochement entre cette remarque et la distinction sexe/genre. L'introduction du nouveau concept de genre laissait espérer plus de clarté et de précisions quant à l'étude de la différence des sexes. Or, après le passage en revue, bien que succinct, de 50 ans de travaux sur le genre, on peut constater que la distinction sexe/genre reste insuffisante et qu'il est nécessaire de rester prudent quant à l'utilisation de celle-ci.

Premièrement, la notion de genre reste vague. On peut remarquer que les différentes conceptions du genre présentées ci-dessus sont étroitement liées à l'orientation théorique des auteurs (parfois avec un certain parti pris). Ainsi, selon les auteurs, le genre est comportemental, identitaire, cérébral, culturel ou sociopolitique.

D'autre part, la notion de genre n'a pas le même degré de pertinence et de validité selon la population étudiée ou le public visé. Pour l'étude des patients intersexués, le genre semble amener un éclaircissement sur leur problématique car le caractère féminin ou masculin de ces personnes entremêle les dimensions psychologique et sociale. Concernant les patients transsexuels et les études du troisième sexe, l'amalgame du genre psychosocial est gênant. Le genre opposé au sexe chez les patients transsexuels est psychologique et non seulement social. Au contraire, chez les personnes du troisième sexe, le genre social semble au premier plan et le genre psychologique est difficile à isoler. Quant au genre dans les mouvements sociopolitiques, d'une part, celui-ci est flou (comme il l'a déjà été constaté ci-dessus). D'autre part, il ne relève dès lors plus que de l'ordre social et de l'influence de la société, ce qui mène à l'amoindrissement voire à la disparition de sa dimension psychologique et du vécu subjectif de l'individu.

\footnotetext{
${ }^{32}$ Freud S. (1920), Sur la psychogénèse d'un cas d'homosexualité féminine in Névrose, psychose et perversion, Paris, PUF, 245-270, 1973.
} 
Dernièrement, il convient de souligner que la distinction sexe/genre a tendance à dériver, à partir de l'opposition entre biologique et social, vers la controverse de l'inné et de l'acquis, vers l'opposition entre nature et culture, délaissant alors dans ces débats le psychologique.

Le genre conçu comme psychosocial mène donc à beaucoup de complexité dans la mesure où il confond le vécu psychologique et le socialement défini. Pour être plus précis, il faudrait faire une nouvelle distinction entre un genre psychologique et un genre social mais cela amoindrirait son objectif de clarté et rendrait plus complexe son étude. De même, l'opposition parfois radicale entre le sexe et le genre a amené à supprimer le sexe. Or, au final, il n'y a pas de genre sans différence des sexes. Par contre, sans la notion de genre, le sexe et la différenciation sexuelle perdurent. Le genre a besoin du sexe pour se définir et même ne peut exister sans lui.

\section{REFERENCES BIBLIOGRAPHIQUES}

Association La revue lacanienne, Melman C. \& al. (2007), Dossier : Sex and gender, Bulletin lacanien, 4, 4, 9-60.

Braconnier A. (2005), Entretien avec Colette Chiland, Carnet psy, 97, 2, 35-41.

Butler J. (1990), Gender trouble : feminism and subversion of identity, New York \& London, trad. franç., Trouble dans le genre : féminisme et subversion de l'identité, Paris, La Découverte, 2005.

Butler J. (2004), La fin de la différence sexuelle in Undoing gender, New York and London, Routledge, trad. franç., Défaire le genre, Paris, Editions Amsterdan, 2006, 201-231.

Califa P. (1997), Retour de manivelle : la transphobie chez les féministes in Sex Changes, The Politics of Transgenderism, San Francisco, Cleis, trad. franc., Le mouvement transgenre, changer de sexe, 123-169, 2003.

Chiland C. (1988), Enfance et transsexualisme, Psychiatrie de l'enfant, XXXI, 2, 313-373.

Chiland C. (1994), Homosexualité féminine et identité sexuée, Revue française de psychanalyse, 1, 147-156.

Chiland C. (1995), De l'essence du féminin, Psychologie clinique et projective, 1, 2, 143-160.

Chiland C. (2003), Nouveaux propos sur la construction de l'identité sexuée, Journal de psychanalyse de l'enfant, 33, 105-122. 
Chiland C. (2005), Problèmes posées aux psychanalystes par les transsexuels, Revue française de psychanalyse, 69, 1, 2, 563-577.

Diamond M. (1965), A critical evaluation of the ontogeny of sexual behavior, Quarterly review of biology, 40, 147-173.

Diamond M. (2000), Sex and gender: same or different?, Feminism \& Psychology, 10 (1), 4654.

Diamond M. \& Sigmundson H. K. (1997), Sex Reassignment at Birth: A Long Term Review and Clinical Implications, Archives of Pediatrics and Adolescent Medicine, 151, 298-304.

Freud S. (1920), Sur la psychogénèse d'un cas d'homosexualité féminine in Névrose, psychose et perversion, Paris, PUF, 245-270, 1973.

Herdt G. (1994), Third Sex, Third Gender, Beyond sexual dimorphism in culture and history, New York, Zone Books.

Laplanche J. (2007), Le genre, le sexe, le sexual in Sexual, la sexualité élargie au sens freudien, Paris, PUF, 153-193.

Money J. Hampson J. G. \& Hampson J. L. (1955), Hermaphrodism : Recommandations concerning assignement of sex, change of sex, and psychologic management, Bulletin of John Hopkins Hospital, 97, 284-300.

Money J., Hampson J. G. \& Hampson J. L. (1957), Imprinting and the establishement of gender role, Archives of neurology and psychiatry, 77, 333-336.

Roscoe W. (1987), Bibliography of berdache and alternative gender roles among North American Indians, Journal of Homosexuality, 14, 3 et 4, 81-171.

Saladin d'Anglure B. (1986), Du fœtus au chamane : la constitution d'un troisième "sexe" inuit, Etudes/Inuit/Studies, 10, 1-2, 25-113.

Stoller R. J. (1968), Sex and gender, vol. 1, New York, Science House, trad. franc. Recherches sur l'identité sexuelle, Paris, Gallimard, 1978.

Stoller R. J. (1985), Presentations of gender, New Haven-London, Yale University Press, trad. franç., Masculin ou féminin ?, Paris, PUF, 1989.

Wittig M. (1973), Le corps lesbien, Paris, Minuit.

\section{Document du web}

David-Ménard M. L'intérêt pour la psychanalyse dans les travaux de Judith Butler : entretien avec Livio Boni in Revue mondiale des francophonies [en ligne]. http://mondesfrancophones.com/espaces/psyches/linteret-pour-la-psychanalyse-dans-lestravaux-de-judith-butler-entretien-avec-livio-bonil (page créée 19 novembre 2007, consultée le 12 novembre 2010). 